\title{
Synchrotron SAXS and WAXS Study of the Interactions of NSAIDs with Lipid Membranes
}

\author{
Cláudia Nunes, ${ }^{\dagger}$ Gerald Brezesinski, ${ }^{\dagger}$ José L. F. C. Lima, ${ }^{\dagger}$ Salette Reis, ${ }^{\dagger}$ and Marlene Lúcio ${ }^{*,+}$ \\ ${ }^{\dagger}$ REQUIMTE, Departamento de Química, Faculdade de Farmácia, Universidade do Porto, Rua Aníbal Cunha, Porto, Portugal \\ ${ }^{\ddagger}$ Max Planck Institute of Colloids and Interfaces, Science Park Golm, Am Mühlenberg 1, 14476 Potsdam, Germany
}

ABSTRACT: Cell membranes often constitute the first biological structure encountered by drugs, and binding or interactions of drugs with lipid components of the membrane may explain part of their mechanism of activity or their side effects. The present study provides evidence of alterations in the structural properties of phospholipid bilayers at acidic conditions that can be correlated with the mechanism of action of nonsteroidal anti-inflammatory drugs (NSAIDs) and with their local action effect on the gastrointestinal tract lipids, aiming a molecular biophysical approach to the interaction of these drugs with lipid membranes. In this context, the structural modifications of the 1,2-dipalmitoyl-sn-glycero-3-phosphocholine bi-
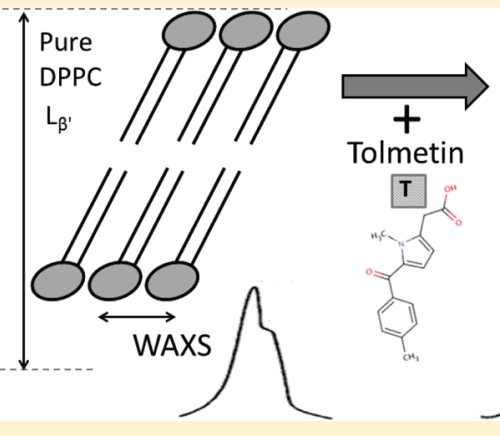
layers at $\mathrm{pH}$ 5.0, induced by increasing concentrations of five NSAIDs (piroxicam, meloxicam, tolmetin, indomethacin, and nimesulide), were studied by small-angle and wide-angle X-ray scattering. Results obtained highlight the effect of each NSAID in modulating the membrane structure properties. All the NSAIDs promoted distinct biophysical effects by perturbing the membrane arrangement to different degrees that are intimately related to their different physicochemical properties as well as with the initial organization of the lipids, depending if they are in the gel $\left(\mathrm{L}_{\beta^{\prime}}\right)$ or in the liquid-crystalline phase $\left(\mathrm{L}_{\alpha}\right)$.

\section{INTRODUCTION}

Nonsteroidal anti-inflammatory drugs (NSAIDs) are recognized for their efficient and incomparable therapeutic effects in numerous chronic and acute conditions. Nevertheless, the toxicity of NSAIDs in the gastrointestinal (GI) tract continues to be a major limitation to their use in the treatment of inflammation and results in thousands of hospitalizations and deaths every year.

The main mechanism of action of NSAIDs is based on their ability to inhibit the enzyme cyclo-oxygenase (COX), which exists in at least two isoforms, COX-1 and COX-2. ${ }^{1}$ The inhibition of COX-1 by the NSAIDs causes the disturbance of prostaglandin synthesis, which is responsible for the protection of the gastric mucosal barrier, maintaining mucosal blood flow, regulating mucosal cell turnover and repair, stimulating secretions of bicarbonate, and developing a protective hydrophobic layer. ${ }^{2}$ Loss of gastric protection by inhibition of prostaglandin synthesis renders the stomach vulnerable to damage by gastric acid, causing mucosal injuries, such as erosions and ulcers, and GI complications, such as bleeding, perforation, and obstruction. ${ }^{3}$ COX-2 selective inhibitors (coxibs) were developed to provide anti-inflammatory and analgesic activity comparable with traditional NSAIDs, but with a lower risk of GI tract injury. ${ }^{4}$ However, the sequence of events resulting from COX inhibition does not completely explain the overall GI toxicity of NSAIDs. ${ }^{5,6}$ In fact, it is described that the NSAIDs can cause GI damage through a variety of different mechanisms, ${ }^{7}$ including the damage of the gastric mucosa by a direct local effect. ${ }^{3}$ In this regard, a hypothesis has been proposed that the NSAIDs compromise the integrity of the gastric mucosa by chemical association with the phospholipids, decreasing the hydrophobic properties of surface mucosal cells predominantly constituted by phosphatidylcholines. ${ }^{8}$ It should be also taken into account that NSAIDs tend to accumulate and reach high concentrations in the gastric mucosa cells, ${ }^{9}$ enhancing the effects of their interaction with the phospholipid lining of the gastric protection barrier.

Many studies have already been performed at physiological $\mathrm{pH}(\mathrm{pH}$ 7.4) and show that NSAIDs are molecules with high membrane partition coefficients ${ }^{10,11}$ that interact strongly with lipid membranes, changing the membrane fluidity ${ }^{12-15}$ and local membrane curvature and thereby conditioning the function of many membrane proteins, enzymes, and receptors. ${ }^{16-18}$ Although NSAID-COX interaction has been well-characterized, the molecular mechanisms underlying the interaction of these drugs with membranes at acidic $\mathrm{pH}$ are still a matter of study. In this context, the current work aims to access the structural modifications arising from the interaction of NSAIDs and lipid membranes, at acidic conditions ( $\mathrm{pH}$ 5.0) by synchrotron small-angle $\mathrm{X}$-ray scattering (SAXS) and wide-angle $\mathrm{X}$-ray scattering (WAXS). To mimic the cellular membrane, liposomes

\footnotetext{
Received: March 16, 2011

Revised: $\quad$ May 17, 2011

Published: May 20, 2011
} 
<smiles>CN1C(=C(O)Nc2ccccc2)C(=O)c2ccccc2S1(=O)=O</smiles><smiles>Cc1cnc(NC(O)=C2C(=O)c3ccccc3S(=O)(=O)N2C)s1</smiles><smiles>COc1ccc2c(c1)c(CC(=O)O)c(C)n2C(=O)c1ccc(Cl)cc1</smiles><smiles>Cc1ccc(C(=O)c2ccc(CC(=O)O)n2C)cc1</smiles><smiles>CS(=O)(=O)Nc1ccc([N+](=O)[O-])cc1Oc1ccccc1</smiles>

Figure 1. Chemical structures and $\mathrm{p} K_{\mathrm{a}}$ values of the NSAIDs investigated: piroxicam (A), meloxicam (B), tolmetin (C), indomethacin (D), and nimesulide (E).

of 1,2-dipalmitoyl-sn-glycero-3-phosphocholine (DPPC) were used. DPPC was the chosen phospholipid once it has been shown that the gastric mucous layer is a protective lipidic membrane in the gel phase composed predominantly of phosphatidylcholines $(30-50 \%)$ with a large amount of the highly surface-active DPPC. $^{19,20}$

With the aim of establishing a correlation between GI injurious actions of NSAIDs and their ability to form a chemical association with phospholipids, the studies included NSAIDs with distinct chemical structures and different selectivities for COX-1 and COX-2 and different reported toxicities. ${ }^{21-23}$ Accordingly, the NSAIDs studied (Figure 1) included tolmetin and indomethacin, which are non-COX-2 selective and have been described as having a high risk of GI toxicity; meloxicam and nimesulide, which show preferential activity toward COX-2 and are better tolerated at the GI tract; and piroxicam, which has been described as a better inhibitor of COX-1 than COX-2 and presents a moderate risk of GI toxicity. ${ }^{24}$

The studies were performed in a range of temperatures from 10 to $70{ }^{\circ} \mathrm{C}$, with the purpose of covering the different lipid phases and assessing the transition temperatures between the phases. Also, static measurements at 20 and $50{ }^{\circ} \mathrm{C}$ were performed to perceive the interactions in the gel and liquidcrystalline phases, respectively.
The results obtained in this work constitute a molecular biophysical approach to the interaction of NSAIDs with lipid membranes at acidic conditions and provide evidence to support the direct local effects of these drugs on the phospholipid lining of the gastric protection barrier.

\section{EXPERIMENTAL SECTION}

Materials. The anti-inflammatory drugs, nimesulide, tolmetin, piroxicam, meloxicam, and indomethacin, were obtained from Sigma-Aldrich, and DPPC was supplied by Avanti Polar-Lipids Inc. These compounds were used without further purification.

All other chemicals were obtained from Merck. Solutions were prepared with water from a Milli-Q plus system with specific conductivity less than $0.1 \mu \mathrm{S} \mathrm{cm}^{-1}$. All the samples were prepared in acetate buffer (0.2 M, pH 5.0)

Lipid Dispersion Preparation. Different amounts of the NSAIDs were mixed with DPPC in a chloroform/methanol mixture $(3: 1 \mathrm{v} / \mathrm{v})$ according to the required molar fraction of the drug $(5,10$, and $20 \mathrm{~mol} \%$ of meloxicam and 20,40 , and 60 $\mathrm{mol} \%$ of the other NSAIDs). Lipid films were formed from these solutions, dried at $50{ }^{\circ} \mathrm{C}$ under a stream of $\mathrm{N}_{2}$, and left overnight under reduced pressure to remove all traces of the organic solvents. 


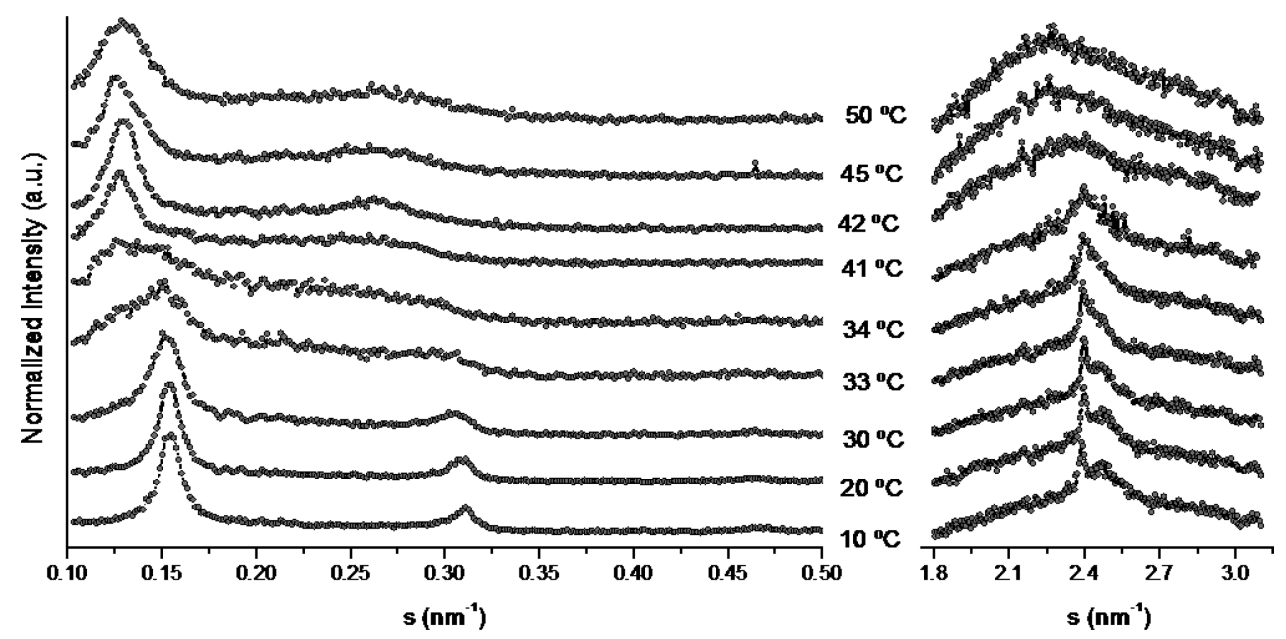

Figure 2. Temperature-dependent small- and wide-angle X-ray diffraction patterns (SAXS and WAXS) of DPPC at pH 5.

The lipid films were hydrated by adding acetate buffer ( $\mathrm{pH}$ 5.0) to reach a $10 \%(\mathrm{w} / \mathrm{v})$ concentration and then alternately heated above the lipid phase transition in a water bath at $60^{\circ} \mathrm{C}$, mixed by vortexing for about $5 \mathrm{~min}$ and centrifuged for $30 \mathrm{~s}$ at $2000 \mathrm{~g}$. This procedure was repeated three times. Finally, the samples were aged overnight at $4{ }^{\circ} \mathrm{C}$ and shaken by vortex at room temperature for $5 \mathrm{~min}$. The dispersions were transferred into glass capillaries, which are transparent for X-rays and $1.5 \mathrm{~mm}$ in diameter (Hilgenberg, Malsfeld, Germany). The flame-sealed capillaries were stored at $4{ }^{\circ} \mathrm{C}$ until the time of the measurements.

X-ray Measurements. SAXS and WAXS experiments were performed at the beamline A2 at Doris III of HASYLAB (DESY, Hamburg, Germany) using a monochromatic radiation with a wavelength of $0.15 \mathrm{~nm}$. The SAXS detector was calibrated with rat-tail tendon (RTT) and the WAXS detector by polyethyleneterephthalat (PET). Heating and cooling scans were performed at a rate of $1{ }^{\circ} \mathrm{C} \mathrm{min}^{-1}$ in the range of $10-70{ }^{\circ} \mathrm{C}$. Data were recorded for $10 \mathrm{~s}$ every minute. Also, static exposures were taken below and above the main transition temperature and compared with the heating/cooling cycles. After each temperature step, the sample has been allowed to equilibrate for $5 \mathrm{~min}$ before the diffraction pattern was recorded. To minimize the X-ray exposure to the sample, a shutter mounted before the sample was kept closed when no data was acquired. Each diffraction pattern is presented as normalized scattering intensity in arbitrary units versus the reciprocal spacing $s(s=(2 \sin \theta) / \lambda$, where $\theta$ is the diffraction angle and $\lambda$ is the $\mathrm{X}$-ray wavelength). The diffraction peaks obtained were fitted with Lorentzians, and the positions of maximum intensities and the full widths of the peaks at one-half of their intensity (fwhm) were determined and used to calculate the correlation length between the lipid bilayers $(\xi=2 \pi /$ fwhm $)$. From the peak maximum positions of the wide- and small-angle diffraction patterns, the repeat distances, $d(d=1 / s)$ were calculated. Errors in experimental values of $d$ and $\xi$ were assessed based on error estimates of the partial molecular volumes of $\mathrm{DPPC}$ and water. ${ }^{25}$

\section{RESULTS AND DISCUSSION}

SAXS and WAXS diffraction spectra obtained for DPPC at $\mathrm{pH}$ 5 and at different temperatures are presented in Figure 2.

The temperatures of the pretransition $\left(\mathrm{L}_{\beta^{\prime}} \rightarrow \mathrm{P}_{\beta^{\prime}}\right)$ and the main phase transition of DPPC $\left(\mathrm{P}_{\beta^{\prime}} \rightarrow \mathrm{L}_{\alpha}\right)$ amount to $33.5 \pm 0.5$
Table 1. Pretransition $\left(T_{\mathrm{p}}\right)$ and Main Transition $\left(T_{\mathrm{m}}\right)$ Temperatures of DPPC and Subsequent Mixtures with $20 \mathrm{~mol} \%$ of Piroxicam, Meloxicam, Tolmetin, Indomethacin, and Nimesulide

\begin{tabular}{lcc} 
& $T_{\mathrm{p}}\left({ }^{\circ} \mathrm{C}\right)\left(\mathrm{L}_{\beta^{\prime}} \rightarrow \mathrm{P}_{\beta^{\prime}}\right)$ & $T_{\mathrm{m}}\left({ }^{\circ} \mathrm{C}\right)\left(\mathrm{P}_{\beta^{\prime}} \rightarrow \mathrm{L}_{\alpha}\right)$ \\
DPPC & $33.5 \pm 0.5$ & $41.5 \pm 0.5$ \\
DPPC + piroxicam & $31.5 \pm 0.5$ & $38.5 \pm 0.5$ \\
DPPC + meloxicam & $31.0 \pm 0.5$ & $40.0 \pm 0.5$ \\
DPPC + tolmetin & & $32.5 \pm 0.5$ \\
DPPC + indomethacin & & $37.5 \pm 0.5$ \\
DPPC + nimesulide & $32.5 \pm 0.5$ & $40.5 \pm 0.5$ \\
\hline
\end{tabular}

and $41.5 \pm 0.5{ }^{\circ} \mathrm{C}$, respectively (Table 1 ). The obtained values are in good agreement with other studies made at physiological $\mathrm{pH} .{ }^{26,27}$

The repeat distance, $d$, deduced from the SAXS patterns of DPPC, increases from $6.54 \pm 0.05 \mathrm{~nm}$ in the $\mathrm{L}_{\beta^{\prime}}$ phase (see also Table 2) to $7.10 \pm 0.05 \mathrm{~nm}$ in the ripple gel phase $\mathrm{P}_{\beta^{\prime}}$. In the $\mathrm{L}_{\beta^{\prime}}$ phase, the hydrophobic chains are tilted (tilt angle is approximately $\left.30^{\circ}\right){ }^{28}$ The loss of the tilt angle would increase the $d$ value by about $0.55 \mathrm{~nm}$. On the other hand, the melting of the nontilted chains would decrease $d$ by about $0.80 \mathrm{~nm}$. Therefore, only a thicker water layer between the lipid bilayers can explain the increase of the repeated distance to $7.46 \pm 0.05 \mathrm{~nm}$ in the $\mathrm{L}_{\alpha}$ phase. The correlation length $(\xi)$ between the bilayers is much lower in the $\mathrm{L}_{\alpha}$ phase compared with the $\mathrm{L}_{\beta^{\prime}}$ phase (Table 2). The correlation length is reduced by the addition of all NSAIDs, highlighting the disturbing effect of these drugs in membrane order. Deconvolution of the WAXS patterns gives two Bragg peaks typical of the pseudohexagonal lattice of the chain packing of DPPC with $4.05 \pm 0.05$ and $4.17 \pm 0.05 \AA$ (Table 3). Increasing temperature leads to a decrease of the lattice distortion (the two Bragg peaks of the orthorhombic lattice come closer together). Moreover, the diffraction peaks present a high correlation length $(\xi)$, which indicates a good correlation between the bilayers. Such a correlation is again reduced by the addition of all NSAIDs, suggesting a disturbance of the membrane in the presence of these drugs.

Comparing the values of $d$ for pure DPPC (in the absence of drugs) obtained in this study at $\mathrm{pH} 5$ (Table 2) with the values obtained at $\mathrm{pH} 7.4,{ }^{29,30}$ it can be seen that the bilayer repeat 
Table 2. Long-Range Distances Determined from SAXS Patterns and Correlation Length $(\xi)$ at 20 and $50{ }^{\circ} \mathrm{C}$ at a $\mathrm{pH}$ Typical of Inflamed Cells ( $\mathrm{pH}$ 5.0 $)^{a}$

\begin{tabular}{|c|c|c|c|c|c|c|c|}
\hline \multirow[b]{2}{*}{ pH 5} & \multirow[b]{2}{*}{$\chi_{\text {DRUG }}$} & \multicolumn{3}{|c|}{$20{ }^{\circ} \mathrm{C}\left(\mathrm{L}_{\beta^{\prime}}\right)$} & \multicolumn{3}{|c|}{$50{ }^{\circ} \mathrm{C}\left(\mathrm{L}_{\alpha}\right)$} \\
\hline & & $d_{1}(\AA)$ & $d_{2}(\AA)$ & $\xi(\AA)$ & $d_{1}(\AA)$ & $d_{2}(\AA)$ & $\xi(\AA)$ \\
\hline DPPC & 0 & $65.4 \pm 0.5$ & & $592 \pm 10$ & $74.6 \pm 0.5$ & & $548 \pm 10$ \\
\hline \multirow[t]{3}{*}{$\mathrm{DPPC}+$ piroxicam } & 20 & $65.1 \pm 0.5$ & & $487 \pm 10$ & $75.0 \pm 0.5$ & $64.3 \pm 0.5$ & $202 \pm 10$ \\
\hline & 40 & $64.7 \pm 0.5$ & & $380 \pm 10$ & $74.9 \pm 0.5$ & $67.6 \pm 0.5$ & $391 \pm 10$ \\
\hline & 60 & $65.1 \pm 0.5$ & & $500 \pm 10$ & $75.8 \pm 0.5$ & $67.0 \pm 0.5$ & $296 \pm 10$ \\
\hline \multirow[t]{3}{*}{ DPPC + meloxicam } & 5 & $65.9 \pm 0.5$ & & $657 \pm 10$ & $71.1 \pm 0.5$ & & $338 \pm 10$ \\
\hline & 10 & $68.1 \pm 0.5$ & & $221 \pm 10$ & $72.1 \pm 0.5$ & & $93 \pm 10$ \\
\hline & 20 & $73.1 \pm 0.5$ & & $263 \pm 10$ & $80.2 \pm 0.5$ & & $36 \pm 10$ \\
\hline \multirow[t]{3}{*}{$\mathrm{DPPC}+$ tolmetin } & 20 & $69.7 \pm 0.5$ & $65.1 \pm 0.5$ & $241 \pm 10$ & $71.1 \pm 0.5$ & $63.9 \pm 0.5$ & $415 \pm 10$ \\
\hline & 40 & $52.1 \pm 0.5$ & & $793 \pm 10$ & $80.7 \pm 0.5$ & & $696 \pm 10$ \\
\hline & 60 & $50.4 \pm 0.5$ & & $89 \pm 10$ & $68.2 \pm 0.5$ & & $89 \pm 10$ \\
\hline \multirow[t]{3}{*}{ DPPC + indomethacin } & 20 & $72.2 \pm 0.5$ & $64.2 \pm 0.5$ & $565 \pm 10$ & $69.4 \pm 0.5$ & $62.6 \pm 0.5$ & $547 \pm 10$ \\
\hline & 40 & $72.9 \pm 0.5$ & $64.8 \pm 0.5$ & $341 \pm 10$ & $69.9 \pm 0.5$ & & $633 \pm 10$ \\
\hline & 60 & $73.3 \pm 0.5$ & $66.8 \pm 0.5$ & $208 \pm 10$ & $70.5 \pm 0.5$ & & $685 \pm 10$ \\
\hline \multirow[t]{3}{*}{ DPPC + nimesulide } & 20 & $65.3 \pm 0.5$ & & $440 \pm 10$ & $76.4 \pm 0.5$ & & $222 \pm 10$ \\
\hline & 40 & $65.4 \pm 0.5$ & & $348 \pm 10$ & $77.3 \pm 0.5$ & & $246 \pm 10$ \\
\hline & 60 & $72.3 \pm 0.5$ & & $316 \pm 10$ & $88.3 \pm 0.5$ & & $232 \pm 10$ \\
\hline
\end{tabular}

Table 3. Short-Range Distances Determined from WAXS Patterns and Correlation Length $(\xi)$ at $20^{\circ} \mathrm{C}$ at a $\mathrm{pH}$ Typical of Inflamed Cells ( $\mathrm{pH} 5.0)^{a}$

\begin{tabular}{lcccr}
\multirow{2}{*}{ pH 5 } & & \multicolumn{3}{c}{$20^{\circ} \mathrm{C}\left(\mathrm{L}_{\beta^{\prime}}\right)$} \\
\cline { 3 - 5 } DPPC & $\chi_{\text {DRUG }}$ & $d_{1}(\AA)$ & $d_{2}(\AA)$ & $\xi(\AA)$ \\
DPPC + piroxicam & 0 & 4.17 & 4.05 & $100 \pm 10$ \\
& 20 & 4.17 & 4.04 & $118 \pm 10$ \\
DPPC + meloxicam & 40 & 4.16 & 4.04 & $104 \pm 10$ \\
& 60 & 4.15 & 4.03 & $89 \pm 10$ \\
DPPC + tolmetin & 5 & 4.18 & 4.07 & $197 \pm 10$ \\
& 20 & 4.17 & 4.02 & $88 \pm 10$ \\
DPPC + indomethacin & 20 & 4.09 & & $36 \pm 10$ \\
& 40 & 4.16 & & $46 \pm \pm 10$ \\
& 60 & 4.16 & & $111 \pm 10$ \\
DPPC + nimesulide & 40 & 4.10 & 3.94 & $52 \pm 10$ \\
& 60 & 4.12 & 4.02 & $47 \pm 10$ \\
& 20 & 4.16 & 4.07 & $213 \pm 10$ \\
& 40 & 4.17 & 4.07 & $220 \pm 10$ \\
& 60 & 4.15 & 4.03 & $89 \pm 10$
\end{tabular}

${ }^{a}$ The data are presented as a function of the mol \% $\left(\chi_{\text {DRUG }}\right)$ of each drug.

distances (measured by SAXS) increase in both phases (gel and liquid-crystalline phases) with the acidic $\mathrm{pH}$. These results suggest that, at $\mathrm{pH} 5$ and at the same temperature, the water layer between the lipid bilayers must be thicker than at $\mathrm{pH}$ 7.4. At $\mathrm{pH} 5$, the DPPC polar groups are slightly positively charged, leading to repulsion between the headgroups and a higher degree of solvation. The larger effective area of the headgroups should lead to a larger tilt angle and, therefore, to a smaller $d$ value. The opposite is observed, and the only reasonable explanation is a thicker water layer between the lipid bilayers.

The diffraction patterns (SAXS and WAXS experiments) of DPPC in acetate buffer ( $\mathrm{pH}$ 5.0) and in acetate buffered solutions with $20 \mathrm{~mol} \%$ of all the drugs at $20{ }^{\circ} \mathrm{C}$ are shown in Figure 3.

When the effect of piroxicam is analyzed, the SAXS measurements show that no significant differences are observed in the gel phase $\left(\mathrm{L}_{\beta^{\prime}}\right)$. The $d$ values remain the same (within the experimental error) even for higher concentrations of the drug, and also, the correlation length is not markedly affected (see also Table 2). Similar to the SAXS region, also in the WAXS region, no significant changes were noticed, showing that the in-plane structure of DPPC is not affected in any way by the presence of piroxicam in the $\mathrm{L}_{\beta^{\prime}}$ phase.

Regarding the transition temperatures of the studied lipid, it was found that, for $40 \mathrm{~mol} \%$ of piroxicam, the lipid pretransition occurs at $31.5 \pm 0.5{ }^{\circ} \mathrm{C}$ and the main phase transition arises at $38.5 \pm 0.5{ }^{\circ} \mathrm{C}$ (Table 1). Therefore, piroxicam lowers both the pretransition and the main phase transition temperatures of the lipid. Relative to the effect of piroxicam at all tested concentrations in the $L_{\alpha}$ phase, SAXS results evidence the existence of an asymmetric Bragg peak indicative of lipid phase separation. A noninfluenced DPPC phase (once the $d$ values are not relevantly changed comparing with pure DPPC) and a mixed DPPC phase with smaller $d$ values (Table 2) coexist. Figure 4, pattern B, illustrates that the very broad asymmetric Bragg peak, corresponding to an influenced and a noninfluenced DPPC gel phase, was observed even at the smaller drug concentration tested (20 mol \%). These observations, as well as the decrease of both phase transition temperatures (Table 1), constitute evidence that piroxicam is able to interact with the lipid even if the membrane disturbing effects induced by this drug do not occur to a high extent when compared to other NSAIDs tested in this work. Furthermore, it is also reasonable to assume that the effects of 

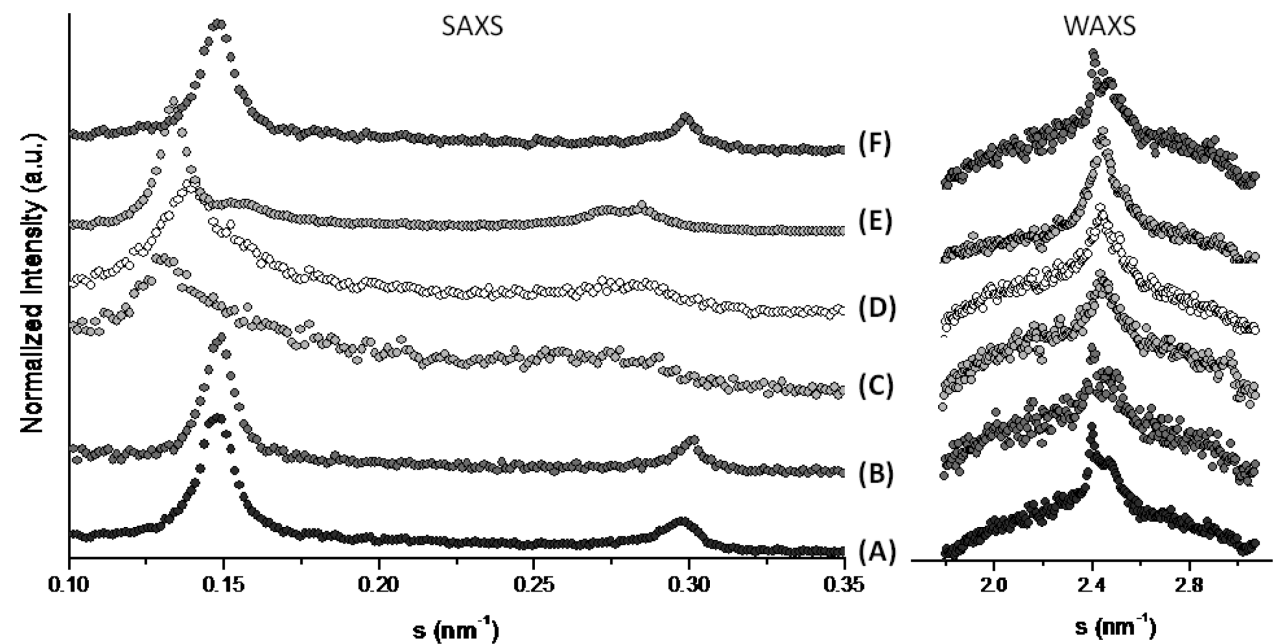

Figure 3. X-ray diffraction patterns (SAXS and WAXS) at $20^{\circ} \mathrm{C}$ for DPPC at $\mathrm{pH} 5.0$ (A) and subsequent mixtures with 20 mol $\%$ of piroxicam (B), meloxicam (C), tolmetin (D), indomethacin (E), and nimesulide (F).

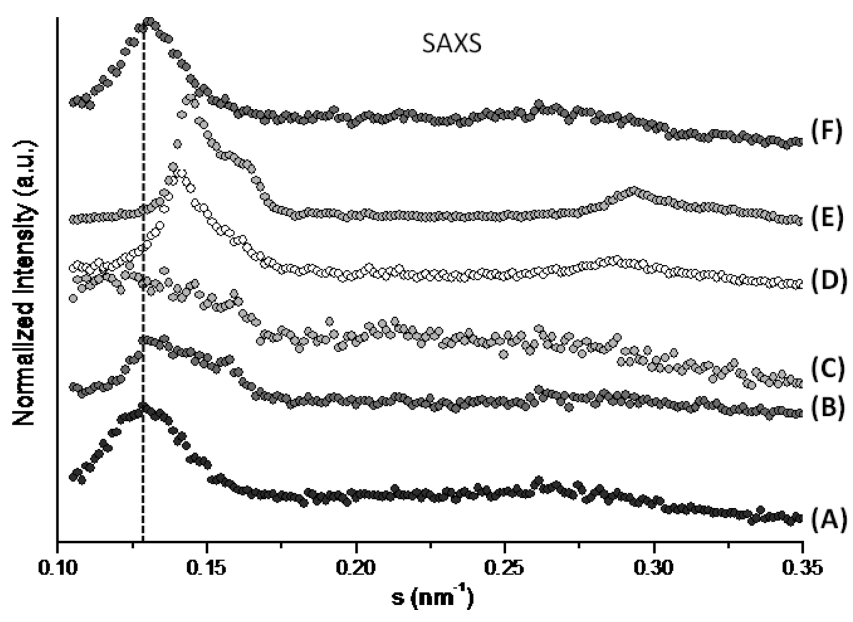

Figure 4. X-ray diffraction patterns (SAXS) at $50{ }^{\circ} \mathrm{C}$ for DPPC at $\mathrm{pH}$ 5.0 (A) and subsequent mixtures with $20 \mathrm{~mol} \%$ of piroxicam (B), meloxicam $(\mathrm{C})$, tolmetin $(\mathrm{D})$, indomethacin $(\mathrm{E})$, and nimesulide $(\mathrm{F})$.

piroxicam are more evident in the $L_{\alpha}$ phase, given the fact that strong interactions between the lipids in the ordered $\mathrm{L}_{\beta^{\prime}}$ phase can hinder a deeper penetration of the drug.

Concerning meloxicam, its presence increases the $d$ values in the $\mathrm{L}_{\beta^{\prime}}$ phase in a concentration-dependent manner (Table 2). At $5 \mathrm{~mol} \%$ of meloxicam, the pretransition of the lipid membrane is strongly affected and occurs at $31.0 \pm 0.5{ }^{\circ} \mathrm{C}$, whereas the main transition arises at $40.0 \pm 0.5{ }^{\circ} \mathrm{C}$ (Table 1 ), which is not significantly different from that of pure DPPC. As the concentration of the drug is increased, the bilayer repeat distances keep rising (Table 2), leading, at $20 \mathrm{~mol} \%$, to values that are characteristic of the $\mathrm{P}_{\beta^{\prime}}$ phase ${ }^{29}$ and to a further reduction of the pretransition temperature. Therefore, at $20{ }^{\circ} \mathrm{C}$, the lipid bilayers containing meloxicam are already in the ripple phase while, at the same temperature, the pure lipid bilayers are still in the $\mathrm{L}_{\beta^{\prime}}$ phase. This consideration is additionally supported by the WAXS measurements. Only one Bragg peak with a low correlation length, described as characteristic of the ripple phase, ${ }^{29}$ was observed in the diffraction patterns. In the $\mathrm{L}_{\alpha}$ phase, meloxicam affects very slightly the long distances up to a concentration of $10 \mathrm{~mol} \%$; however, as the concentration increases $(20 \mathrm{~mol} \%)$, the effect of this drug on the lipid bilayers becomes very evident, strongly increasing the $d$ value to $80.2 \pm$ $0.5 \mathrm{~nm}$ (Table 2). This concentration-dependent effect might be explained by the fact that meloxicam, belonging to the oxicams' chemical group of NSAIDs, is predominantly in the anionic form at $\mathrm{pH} 5$ due to the deprotonation of the enolic $\mathrm{OH}$ (see the $\mathrm{p} K_{\mathrm{a}}$ of meloxicam in Figure 1). ${ }^{31,32}$ However, oxicams are described to be prototropic compounds ${ }^{32}$ that may modify their ionization state depending on the microenvironment or on the amount of water in the surrounding. For low drug concentrations, the waterto-drug ratio is sufficiently high to support the formation of anion in solution, but as the concentration of the drug increases, the water-to-drug ratio decreases and most of the drug molecules face a relatively apolar environment, which determines the formation of neutral forms. ${ }^{32}$ Therefore, at higher concentrations of meloxicam, there is a predominance of the neutral form, which is, in turn, more prone to penetrate into the membrane and perturb the lipid bilayer, justifying the higher effect on the $d$ values found with increasing drug concentration. It is also important to refer to the fact that piroxicam is also a member of the oxicam group of NSAIDs. Nonetheless, meloxicam has a higher affinity for the hydrophobic environment of lipids than piroxicam. ${ }^{33}$ This differential behavior may be attributed to the difference in their chemical structures, particularly to the existence of a side methyl substituted ring instead of a pyridine group in meloxicam (see Figure 1). Indeed, the existence of this methyl side chain in meloxicam has been considered as responsible for an increased membrane penetration and may justify the enhanced membrane perturbing effect observed for this drug. ${ }^{33} \mathrm{It}$ should be also noticed that the presented measurements for meloxicam are made with smaller molar fractions than for all the other NSAIDs; for higher molar fractions, the diffraction peaks almost disappear, meaning that this NSAID induces a high perturbation of the bilayer order. In addition, the drug/lipid molar fractions studied were intentionally high to mimic the actual concentrations resulting from drug-lipid interactions that lead to drug accumulation and, therefore, to much higher concentrations of drugs in the lipid membranes than in the aqueous phase. ${ }^{34}$ 

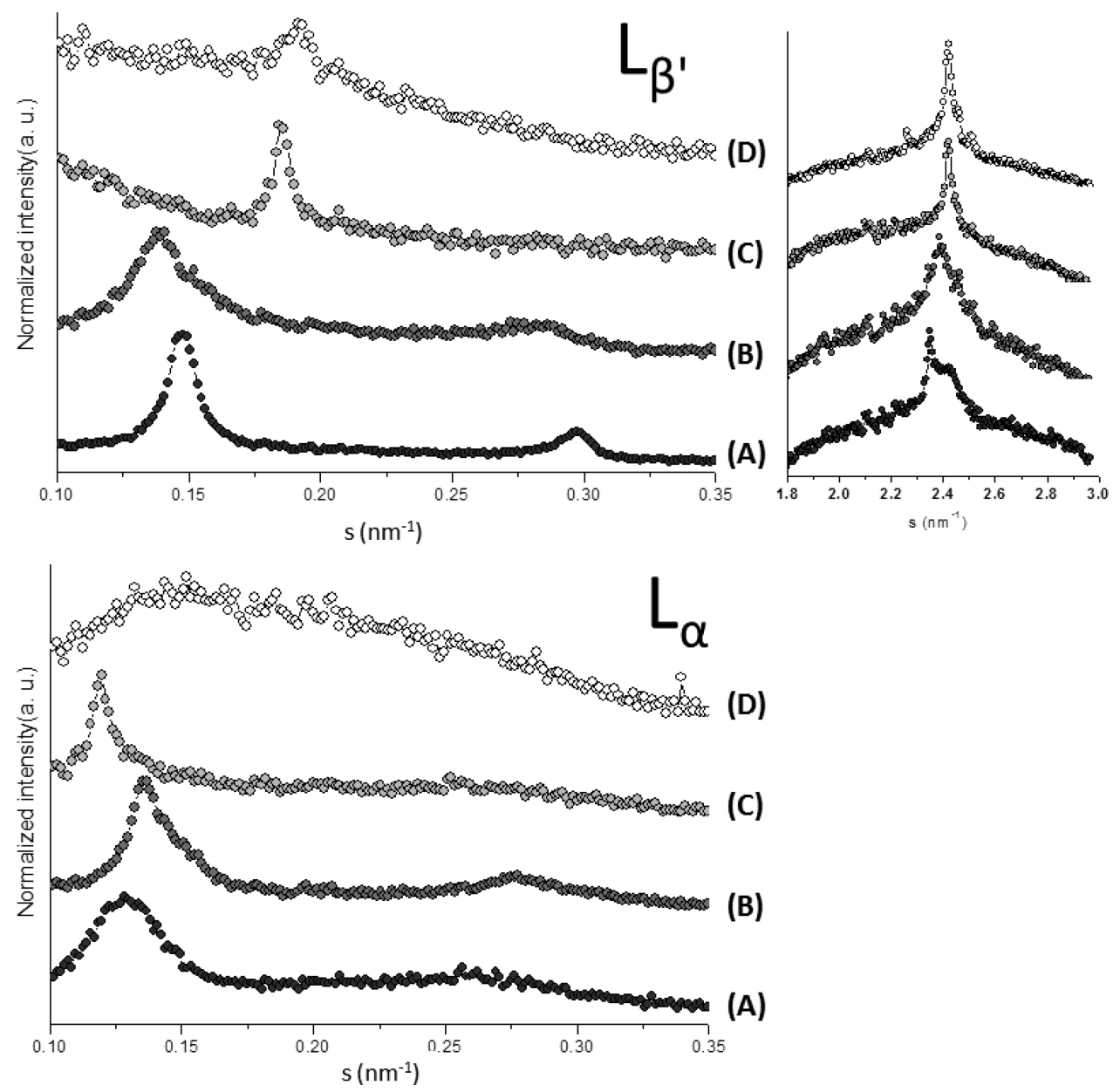

Figure 5. X-ray diffraction patterns (SAXS and WAXS) at 20 and $50{ }^{\circ} \mathrm{C}$ for DPPC at $\mathrm{pH} 5.0$ and subsequent mixtures with tolmetin at different concentrations ( $\mathrm{mol} \%): 0$ (A), $20(\mathrm{~B}), 40(\mathrm{C})$, and $60(\mathrm{D})$.

As in the case of meloxicam, the interaction of tolmetin in each lipid phase is drug/lipid ratio-dependent. In the $\mathrm{L}_{\beta^{\prime}}$ phase, the presence of tolmetin in a small molar fraction increases the $d$ values for the long distances, and at higher concentrations, the values decrease to much smaller values than for pure DPPC. Diffraction patterns for all phases and tolmetin/lipid ratios are presented in Figure 5.

For the smaller molar fraction of tolmetin $(20 \mathrm{~mol} \%)$, it is possible to deconvolute two peaks from the first-order diffraction: one that corresponds to a noninfluenced phase (because it presents a similar value of $d$ as pure DPPC; see Table 2) and another one with a higher correlation length that corresponds to the lipid phase influenced by tolmetin.

At $\mathrm{pH} 5$, tolmetin is almost completely negatively charged, because this drug presents a $\mathrm{p} K_{\mathrm{a}}$ of $3.5^{35}$ and the acidic carboxyl group is, therefore, deprotonated at the $\mathrm{pH}$ of the studies (Figure 1). Thus, for low percentages of drug, the negatively charged tolmetin may partially penetrate within the polar region of the phospholipids, hence enhancing the membrane disorganization. This is also consistent with the results of WAXS measurements in which a dislocation of both Bragg peak positions as well as a diminished correlation length can be seen. However, for higher concentrations of tolmetin, the high density of negative charges putatively establishes strong electrostatic bonds with the positively charged choline headgroup. In fact, in the literature, it can be found that very strong electrostatic bonds as well low $\mathrm{pH}$ conditions favor interdigitation of the lipid bilayers. $^{36}$ The interaction of tolmetin with the polar headgroups of the lipid bilayer, especially at higher drug concentrations ( $40 \mathrm{~mol} \%$ ), is further confirmed by DSC experiments showing the abolishment of the pretransition peak, ${ }^{13,37}$ meaning that, in the presence of tolmetin, the lipid does not form the ripple phase. Indeed, it has been observed by differential calorimetric measurements that the existence of the pretransition is a consequence of the rotation of the phospholipid headgroups or of conformational changes in the phospholipid bilayer structure. Any compound that interacts with the headgroups will affect or abolish the pretransition. ${ }^{37}$ Additionally, tolmetin also affects the main transition temperature of the lipid that, in the presence of this NSAID, decreases to $32.5 \pm 0.5{ }^{\circ} \mathrm{C}$ (Table 1).

By the presence of higher concentrations of tolmetin, the WAXS profiles show the existence of a sharp reflection typical of the hexagonal packing of nontilted chains. ${ }^{38,39}$ The change from the orthorhombic unit cell with tilted chains to the hexagonal packing of nontilted chains by reducing the area requirement of the headgroups would lead to an increase of the bilayer thickness. However, SAXS measurements indicate a reduction of the $d$ values. The only explanation for a simultaneous decrease of the tilt angle and a decrease of the bilayer thickness would be the penetration of the drug into the headgroup region, thereby 
increasing the area requirement of the headgroups and leading to an interdigitation of the acyl chains to decrease the mismatch of area requirements between heads and tails (see Figure 6). Such an interdigitation of the acyl chains is energetically more favorable than a strong tilting (exceeding a threshold value) of the chains.

In the $\mathrm{L}_{\alpha}$ phase, the membrane is naturally more disordered and there is more space between the polar headgroups of the

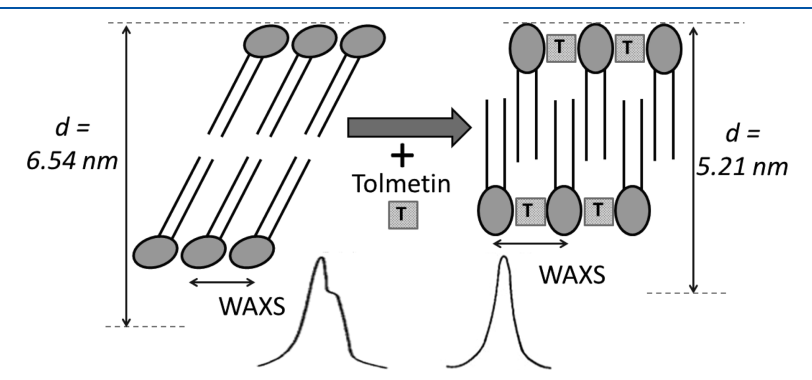

Figure 6. Schematic representation of the interaction of $40 \mathrm{~mol} \%$ of tolmetin with the phospholipid bilayer at the $\mathrm{L}_{\beta^{\prime}}$ phase. phospholipids in which tolmetin can be located, and thus, in this phase, tolmetin presents the same effect as in the $\mathrm{L}_{\beta^{\prime}}$ phase, although to a smaller extent.

In the $\mathrm{L}_{\beta^{\prime}}$ phase, all molar fractions of indomethacin increase the $d$ values, approximately to the same value (Table 2 ). It should be noticed that, for $20 \mathrm{~mol} \%$, two peaks are observed: one at $s=$ $0.138 \mathrm{~nm}$ and a smaller one at $0.155 \mathrm{~nm}$ (Figure 7). This means that the drug is not homogenously distributed in the membrane, but there is an influenced phase corresponding to the first peak and a noninfluenced phase corresponding to the second peak, which is approximately at the same position as that of the pure DPPC phase. Thus, a higher concentration of indomethacin is needed to affect the whole membrane to the same extent. Similar to tolmetin, indomethacin is also a weak acid $\left(\mathrm{p} K_{\mathrm{a}}=4.5\right)^{35}$ and presents a deprotonated carbonyl group negatively charged at the $\mathrm{pH}$ of the studies (Figure 1). The ionized carbonyl group determines the interaction of indomethacin with the polar headgroups of the phospholipids with a consequent decrease in the tilt angle, which leads to a thicker bilayer (increase of the $d$ values). Once the increase of the distance is superior to $0.55 \mathrm{~nm}$ (characteristic distance due to the loss of the tilt angle), it can be

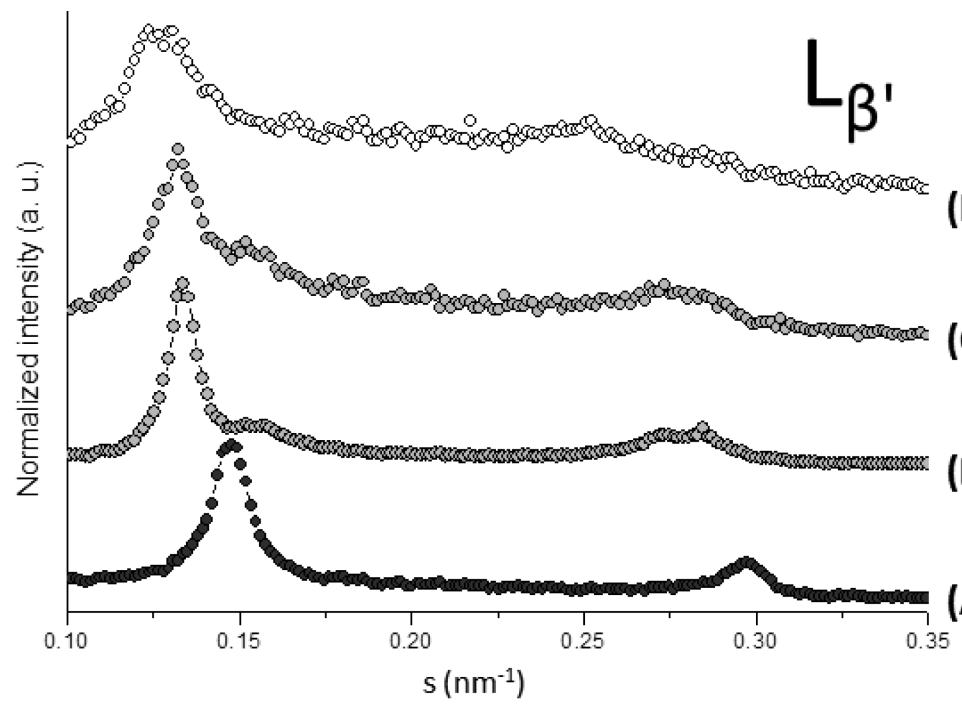

(D)
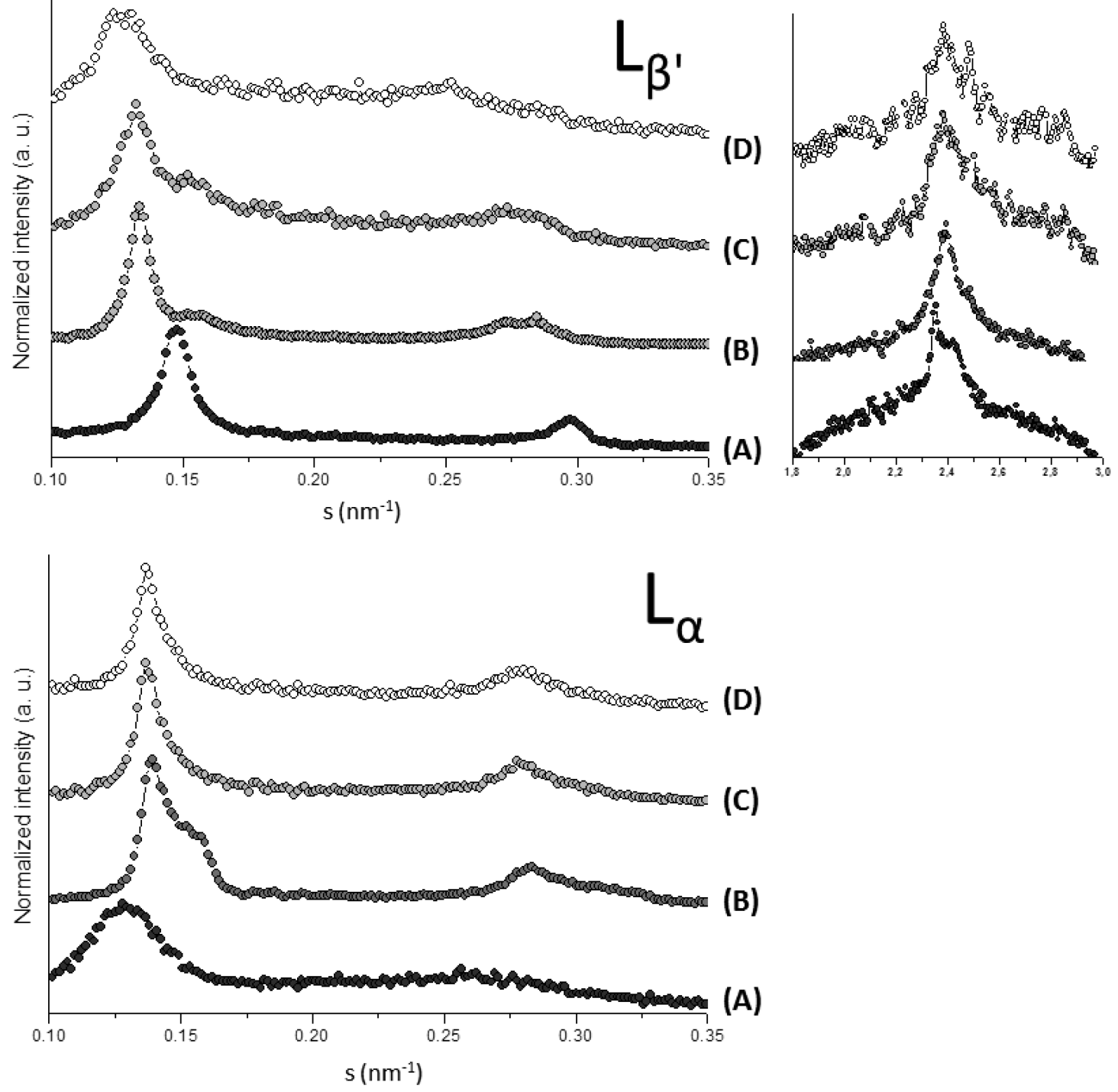

Figure 7. X-ray diffraction patterns (SAXS and WAXS) at 20 and $50{ }^{\circ} \mathrm{C}$ for DPPC at $\mathrm{pH} 5.0$ and subsequent mixtures with indomethacin at different concentrations (mol \%): 0 (A), 20 (B), 40 (C), and 60 (D). 
assumed that there is also an increase of the water layer. Regarding the measurements in the WAXS region, the distances of the two Bragg peaks decrease, which shows that the lattice distortion is reduced by approaching a hexagonal packing (progressive decrease of the tilt angle) as the drug penetration between the polar headgroups takes place (Table 3).

The interaction of indomethacin with the membrane also prevents the formation of the ripple phase, leading directly to the main phase transition from the $\mathrm{L}_{\beta^{\prime}}$ to the $\mathrm{L}_{\alpha}$ phase at a decreased temperature of $37.5 \pm 0.5{ }^{\circ} \mathrm{C}$ (Table 1). This is in agreement with previously reported DSC studies where indomethacin has also shown to abolish the pretransition peak. ${ }^{28}$

In the $\mathrm{L}_{\alpha}$ phase, the $d$ values of DPPC decrease in the presence of indomethacin compared with the pure DPPC (Table 2), which can be explained by a partially interdigitated phase, which has already been observed in other experiments at physiological $\mathrm{pH} .{ }^{30}$ Again and only for the smallest molar fraction analyzed (20 $\mathrm{mol} \%)$, there is a coexistence of two phases (corresponding to two $d$ values), meaning that the bilayer is not homogenously affected by the presence of the drug.

In the case of nimesulide and concerning both phases, its effect is only noticed for the highest molar fraction. Nimesulide is almost neutral at the $\mathrm{pH}$ of this study ${ }^{40}$ and is able to interact with both the polar headgroups and the hydrophobic acyl chains, as seen by the decrease of both the pre- and the main transition temperatures to $32.5 \pm 0.5$ and $40.5 \pm 0.5{ }^{\circ} \mathrm{C}$, respectively (Table 1). However, despite being able to penetrate into the lipid bilayers, nimesulide does not perturb the lipid packing to a great extent. This might be related to a certain planarity of the nimesulide molecule, being able to interpenetrate between the phospholipids without inducing noticeable perturbation.

\section{CONCLUSIONS}

The principal targets of NSAIDs in the control of pain and inflammation are the membrane-associated enzymes COX. To bind with their targets, NSAIDs have to pass through the membranes, and thus, the interactions of these drugs with the lipids of the biomembranes are expected to play a major role in guiding their COX inhibition. Evidence has accumulated during the past decade to support the view that the capacity of NSAIDs to interact with lipid membranes contributes not only decisively to their therapeutic effects but also to the type and incidence of their toxic side effects. In fact, it has been shown that NSAIDs reach particularly high concentrations in compartments in which they cause therapeutic and side effects, and the accumulation within gastric mucosal cells is a principal factor associated with the intervention of intracellular biochemical events and resultant gastric mucosal damage. ${ }^{9}$ However, it has also been shown that prostaglandins, synthesized locally by COX-1 in the gastric mucosa, play a physiologically important role in protecting the tissue against damaging agents, such as acid. ${ }^{41}$ Therefore, it can be said that there is not only one mechanism responsible for the NSAIDs toxicity, but there are two important mechanisms that may condition the resultant gastric toxicity of these drugs, which are the inhibition of COX-1 with the consequent inhibition of the gastroprotective prostaglandins and the local action of NSAIDs in the lipid gel layer that protects gastric mucosa.

The present study supports the role played in GI toxic effects by the local interactions of high concentrations of NSAIDs (typical of chronic administrations) with acidic phospholipids. The local effect of NSAIDs in the lipid GI protective layer is herein evaluated by the ability of these drugs to form a chemical association with phospholipids in a lipid model system that includes the main constituent of biological membranes, in particular, of the gastric mucosa (DPPC). Furthermore, because the local effect of NSAIDs on the lipid protective layer of the gastric mucosa does not completely explain the overall gastric toxicity of these drugs (which is also related to their capacity of inhibiting COX-1), the studies performed included a wide range of NSAIDs with distinct chemical structures and different selectivities for COX-1 and COX-2 and different reported toxicities (COX-2 selective drugs would be expected to present less GI toxicity). The GI toxicity of the NSAIDs studied will be thus correlated both with the results of the drug-lipid interaction evaluated within this work and with the literature information regarding the selectivity of the NSAIDs studied toward COX-2.

Meloxicam is considered to be one of the better-tolerated NSAIDs, and this has been associated with the fact that meloxicam is a potent selective inhibitor of COX-2. Although, in the current work, its effect on the membrane structure has been shown to be superior to the one of piroxicam, this local perturbing effect of the lipid membrane is probably balanced by the selectivity toward COX-2, making meloxicam a very effective and safe drug because the therapeutic benefits are combined with good gastrointestinal tolerability. The present study is, however, coherent with the loss of GI tolerability found for meloxicam at high local gastric concentrations. Indeed, it has been reported that meloxicam shows GI injuries at high doses similar to the ones provoked by piroxicam, and it has been speculated that meloxicam might lose COX-2 selectivity at higher doses. ${ }^{22}$ It must also be the case that both a local effect in the GI protector phospholipids and the COX-2 selectivity concur for meloxicam gastric toxicity. On one hand, low doses of meloxicam have less direct effects on GI phospholipids, which are balanced by its selectivity to COX-2. On the other hand, if the concentration increases, the direct perturbing effects of meloxicam on membrane phospholipids lead to protective layer disruption, and this interaction with the phospholipids (also observed in this work) is possibly too strong to be balanced by the COX-2 selectivity of this drug.

In the case of nimesulide, its GI tolerability is correlated with both its COX-2 selectivity and its low local effect in the GI protector phospholipids also observed in the current study.

Piroxicam belongs to the same enolic acid group of the oxicam class of NSAIDs as meloxicam. Notwithstanding the chemical similarity of these two drugs, meloxicam has been estimated to have a 3 - to 77 -fold greater affinity for COX-2, ${ }^{42,43}$ and for that reason, piroxicam is less well GI tolerated, even presenting less local lipid perturbation effects.

The great interaction of indomethacin with the membrane observed in the current work associated with the lack of selectivity toward COX-2 turns this drug into one of the most toxic to the GI tract. The same kind of reasoning can be established for tolmetin, which revealed a strong interaction with the headgroups of the phospholipid bilayer in both lipid phases. Particularly, its great effect in the lipid gel phase can explain its GI toxicity, which limits its application to endovenous routes of administration and in postchirurgic stages.

\section{AUTHOR INFORMATION}

Corresponding Author

*Tel: +351-222078966. Fax: +351-222078961.E-mail: mlucio@ ff.up.pt. 


\section{ACKNOWLEDGMENT}

C.N. thanks FCT for the doctoral grant (SFRH/BD/38445/ 2007). The authors thank HASYLAB at DESY, Hamburg, Germany, for beam time and support through the project I-20080033 EC. The authors are grateful to Dr. Sérgio Fungari for help at beamline $\mathrm{A} 2$.

\section{REFERENCES}

(1) Vane, J. R.; Botting, R. M. Am. J. Med. 1998, 104, 2S.

(2) Hayllar, J.; Bjarnason, I. Lancet 1995, 346, 521.

(3) Hawkins, C.; Hanks, G. W. J. Pain Symptom Manage. 2000, 20,140 .

(4) Coruzzi, G.; Venturi, N.; Spaggiari, S. Acta Biomed. 2007, 78, 96.

(5) Sostres, C.; Gargallo, C. J.; Arroyo, M. T.; Lanas, A. Best Pract. Res., Clin. Gastroenterol. 2010, 24, 121.

(6) Mahmud, T.; Scott, D. L.; Bjarnason, I. Ann. Rheum. Dis. 1996, 55, 211.

(7) Brzozowski, T. Clin. Exp. Pharmacol. Physiol. 2010, 37, 651.

(8) Khazaeinia, T.; Jamali, F. J. Pharm. Pharm. Sci. 2003, 6, 351.

(9) Mccormack, K.; Brune, K. Arch. Toxicol. 1987, 60, 261.

(10) Lucio, M.; Ferreira, H.; Lima, J. L.; Reis, S. Med. Chem. 2006, $2,447$.

(11) de Castro, B.; Gameiro, P.; Lima, J. L. F. C.; Matos, C.; Reis, S. Colloids Surf., A 2001, 190, 205.

(12) Sousa, C.; Nunes, C.; Lucio, M.; Ferreira, H.; Lima, J. L. F. C.; Tavares, J.; Cordeiro-Da-Silva, A.; Reis, S. J. Pharm. Sci. 2008, 97, 3195.

(13) Lucio, M.; Nunes, C.; Gaspar, D.; Golebska, K.; Wisniewski, M.; Lima, J. L. F. C.; Brezesinski, G.; Reis, S. Chem. Phys. Lett. 2009, 471, 300.

(14) Czapla, K.; Korchowiec, B.; Rogalska, E. Langmuir 2009, 26, 3485.

(15) Wieclaw, K.; Korchowiec, B.; Corvis, Y.; Korchowiec, J.; Guermouche, H.; Rogalska, E. Langmuir 2009, 25, 1417.

(16) Lichtenberger, L. M.; Wang, Z. M.; Romero, J. J.; Ulloa, C.; Perez, J. C.; Giraud, M. N.; Barreto, J. C. Nat. Med. 1995, 1, 154.

(17) Gaspar, D.; Lucio, M.; Rocha, S.; Lima, J. L.; Reis, S. Chem. Phys. Lipids 2011.

(18) Gaspar, D.; Lucio, M.; Wagner, K.; Brezesinski, G.; Rocha, S.; Costa Lima, J. L.; Reis, S. Biophys. Chem. 2010, 152, 109.

(19) Mosnier, P.; Rayssiguier, Y.; Motta, C.; Pelissier, E.; Bommelaer, G. Gastroenterology 1993, 104, 179.

(20) Allen, A.; Pearson, J. P. The Gastrointestinal Adherent Mucous Gel Barrier. In Glycoprotein Methods and Protocols: The Mucins; Corfield, A. P., Ed.; Humana Press: Totowa, NJ, 2000; Vol. 125, p 57.

(21) McCarthy, D. M. Am. J. Med. 1999, 107, 37 S.

(22) Hawkey, C. J. Best Pract. Res., Clin. Gastroenterol. 2001, 15, 801.

(23) Giuliano, F.; Ferraz, J. G.; Pereira, R.; de Nucci, G.; Warner,

T. D. Eur. J. Pharmacol. 2001, 426, 95.

(24) Frolich, J. C. Trends Pharmacol. Sci. 1997, 18, 30.

(25) Greenwood, A. I.; Tristram-Nagle, S.; Nagle, J. F. Chem. Phys. Lipids 2006, 143, 1.

(26) Wang, Y.; Dea, P. J. Chem. Eng. Data 2009, 54, 1447.

(27) Reeves, M. D.; Schawel, A. K.; Wang, W. D.; Dea, P. Biophys. Chem. 2007, 128, 13.

(28) Lucio, M.; Bringezu, F.; Reis, S.; Lima, J. L. F. C.; Brezesinski, G. Langmuir 2008, 24, 4132.

(29) Winter, R. High Pressure Effects in Molecular Bioscience. In Chemistry at Extreme Conditions, 1a ed.; Manaa, M. R., Ed.; Elsevier B.V.: Amsterdam, 2004; p 29.

(30) Nunes, C.; Brezesinski, G.; Lima, J. L. F. C.; Reis, S.; Lucio, M. Soft Matter 2011, 7, 3002.

(31) Banerjee, R.; Chakraborty, H.; Sarkar, M. Spectrochim. Acta, Part A 2003, 59, 1213.

(32) Banerjee, R.; Sarkar, M. J. Lumin. 2002, 99, 255.

(33) Kyrikou, I.; Hadjikakou, S. K.; Kovala-Demertzi, D.; Viras, K.; Mavromoustakos, T. Chem. Phys. Lipids 2004, 132, 157.
(34) Wiseman, H. J. Nutr. Biochem. 1996, 7, 2.

(35) Osterberg, T.; Svensson, M.; Lundahl, P. Eur. J. Pharm. Sci. 2001, 12, 427.

(36) Furuike, S.; Levadny, V. G.; Li, S. J.; Yamazaki, M. Biophys. J. 1999, 77, 2015.

(37) Biruss, B.; Dietl, R.; Valenta, C. Chem. Phys. Lipids 2007, $148,84$.

(38) Zantl, R.; Baicu, L.; Artzner, F.; Sprenger, I.; Rapp, G.; Radler, J. O. J. Phys. Chem. B 1999, 103, 10300.

(39) Tang, P.; Eckenhoff, R. G.; Xu, Y. Biophys. J. 2000, 78, 1804.

(40) Rainsford, K. D. Inflammopharmacology 2006, 14, 120.

(41) Lichtenberger, L. M.; Graziani, L. A.; Dial, E. J.; Butler, B. D.; Hills, B. A. Science 1983, 219, 1327.

(42) Patrignani, P.; Panara, M. R.; Sciulli, M. G.; Santini, G.; Renda, G.; Patrono, C. J. Physiol. Pharmacol. 1997, 48, 623.

(43) Cryer, B.; Dubois, A. Prostaglandins Other Lipid Mediators 1998, 56,341 . 